\title{
Two-Photon Absorption and Self-Phase Modulation in Silicon Optical Fibers into the Mid-Infrared Regime
}

\author{
L. Shen ${ }^{1}$, N. Healy ${ }^{1}$, T. D. Day ${ }^{2}$, J. R. Sparks ${ }^{2}$, J. V. Badding ${ }^{2}$, and A. C. Peacock ${ }^{1}$ \\ 1. Optoelectronics Research Centre, University of Southampton, Southampton SO17 1BJ, United Kingdom \\ 2. Department of Chemistry and Materials Research Institute, Pennsylvania State University 16802 PA, USA \\ acp@orc.soton.ac.uk
}

\begin{abstract}
Nonlinear transmission is investigated in a hydrogenated amorphous silicon optical fiber extending into the mid-infrared region. Low losses past the two-photon absorption edge allow for strong spectral broadening in this important wavelength regime.

OCIS codes: (060.2270) Fiber characterization; (060.2290) Fiber materials; (060.4370) Nonlinear optics.
\end{abstract}

Nonlinear silicon photonics is a rapidly growing area of research with applications ranging from optical switching to photon pair generation. More recently, hydrogenated amorphous silicon (a-Si:H) has become an increasingly popular material for nonlinear silicon photonics due to its low transmission losses, high Kerr nonlinearity and low fabrication costs [1]. To date, this material has largely been characterized at telecoms wavelengths where the nonlinear losses are expected to be lower than crystalline silicon (due to the larger bandgap and hence the shorter two-photon absorption cut-off wavelength), but yet these have still been shown to be non-negligible [1,2]. In this paper we extend our nonlinear investigations of the a-Si:H core fibers to present the first characterization of both the TPA parameter $\beta_{\text {TPA }}$ and the Kerr nonlinear index $n_{2}$ over a wavelength region that crosses the TPA edge. The low optical losses approaching $2 \mu \mathrm{m}$ allow for strong spectral broadening indicating the suitability for these fibres to find applications in the mid-infrared regime.

Our silicon core fibers are fabricated using the well-established high pressure microfluidic chemical technique to deposit a-Si:H material into the central hole of a silica capillary [2]. The fiber used in our characterization has a core diameter of $\sim 6 \mu \mathrm{m}$ and a linear loss starting at $2.5 \mathrm{~dB} / \mathrm{cm}$ for $1540 \mathrm{~nm}$ and decreasing to $1.5 \mathrm{~dB} / \mathrm{cm}$ at $2000 \mathrm{~nm}$. The bandgap energy of amorphous silicon is known to be around $E_{g} \sim 1.7 \mathrm{eV}$. To characterize $\beta_{\text {TPA }}$ across the TPA edge $\left(E_{g} / 2\right)$ we used a Ti:sapphire pumped OPO with $200 \mathrm{fs}$ pulses at $80 \mathrm{MHz}$, tuned from $1400 \mathrm{~nm}$ to $2200 \mathrm{~nm}$. By measuring the output power as a function of the coupled input power, $\beta_{\mathrm{TPA}}$ can be obtained from the slope of the inverse of the transmission given by $1 / T=P_{\text {in }} / P_{\text {out }}[1]$. The corresponding values are plotted as a function of wavelength in Fig. 1(a) with the results showing that $\beta_{\text {TPA }}$ drops rapidly as it approaches $1750 \mathrm{~nm}$, indicating a bandgap energy for our material of $\sim 1.4 \mathrm{eV}$. Following this, it is useful to characterize the nonlinear spectral broadening for wavelengths both above and below the estimated TPA edge. Figs. 1(b) and (c) show the spectral broadening due to self-phase modulation at $1540 \mathrm{~nm}$ and $1850 \mathrm{~nm}$, respectively. It is clear that whilst both spectra exhibit significant broadening at high pump powers, a slightly larger bandwidth of $\sim 250 \mathrm{~nm}$ is obtained at $1850 \mathrm{~nm}$ compared to $\sim 80 \mathrm{~nm}$ at $1540 \mathrm{~nm}$. By fitting the experimental spectral broadening using a modified form of the Schrödinger equation we have previously characterized $n_{2}$ to be $\sim 1.8 \times 10^{-13} \mathrm{~cm}^{2} / \mathrm{W}$ at $1540 \mathrm{~nm}$, which is more than twice that of crystalline silicon [2]. Using similar analysis we will characterize the values of $n_{2}$ for our a-Si:H core material across the full wavelength region and determine the relative nonlinear figure of merit of these fibers in both the near and mid-infrared regions.
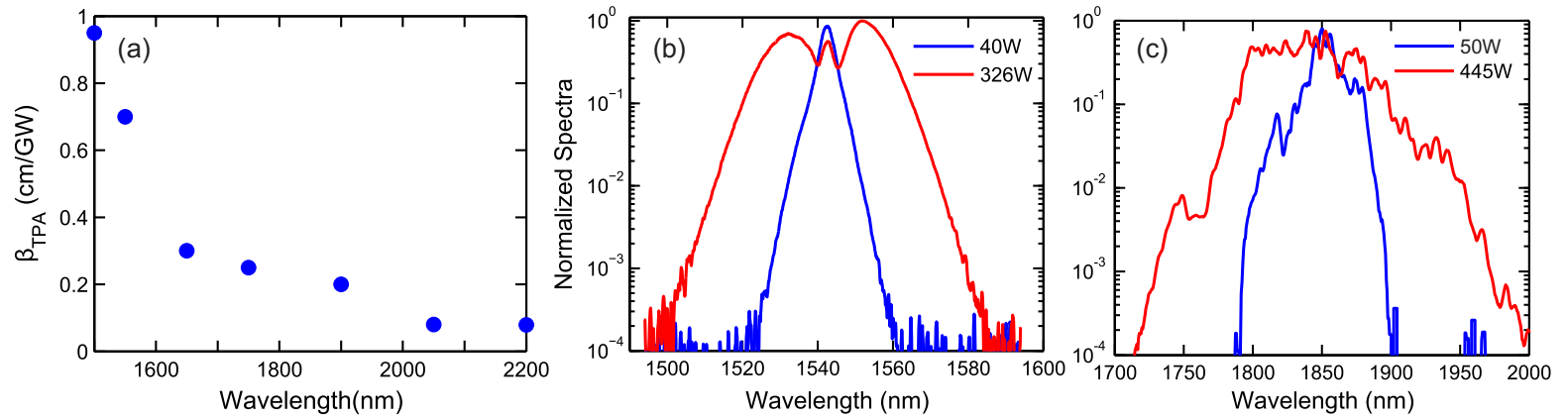

Fig. 1. (a) TPA parameter as a function of wavelength. Spectral broadening for (b) $1540 \mathrm{~nm}$ and (c) $1850 \mathrm{~nm}$, input peak powers given in the legend.

\section{References}

1. B. Kuyken, H. Ji, S. Clemmen, S. K. Selvaraja, H. Hu, M. Pu, M. Galili, P. Jeppesen, G. Morthier, S. Massar, L.K. Oxenlwe, G. Roelkens, and R. Baets, "Nonlinear properties of and nonlinear processing in hydrogenated amorphous silicon waveguides," Opt. Express 19, B146-B153 (2011).

2. P. Mehta, N. Healy, N. F. Baril, P. J. A. Sazio, J. V. Badding, and A. C. Peacock, "Nonlinear transmission properties of hydrogenated amorphous silicon core optical fibers," Opt. Express 16, 16826-16831 (2010). 